\title{
Protein Denaturation, Zero Entropy Temperature, and the Structure of Water around Hydrophobic and Amphiphilic Solutes
}

Supporting Information

\author{
Kazimieras Tamoliūnas ${ }^{\mathrm{a}}$, Nuno Galamba ${ }^{\mathrm{a}, *}$ \\ ${ }^{a}$ Centre of Chemistry and Biochemistry and Biosystems and Integrative Sciences Institute, Faculty of Sciences of the \\ University of Lisbon, C8, Campo Grande, 1749-016 Lisbon, Portugal.
}

${ }^{*}$ Corresponding author. Electronic mail: njgalamba@fc.ul.pt 
Table S1 - Hydration free energy at $298 \mathrm{~K}$ and 1 atm for various nonpolar and polar OPLS-aa model solutes in different water models.

\begin{tabular}{cccccc}
\hline & TIP3P & SPC/E & TIP4P-Ew & TIP4P-2005 & Experimental \\
& $\Delta G_{\mathrm{hyd}}\left(\mathrm{kJmol}^{-1}\right)$ & $\Delta G_{\mathrm{hyd}}\left(\mathrm{kJmol}^{-1}\right)$ & $\Delta G_{\mathrm{hyd}}\left(\mathrm{kJmol}^{-1}\right)$ & $\Delta G_{\mathrm{hyd}}\left(\mathrm{kJmol}^{-1}\right)$ & $\Delta G_{\mathrm{hyd}}\left(\mathrm{kJmol}^{-1}\right)$ \\
\hline Methane & $+9.1 \pm 0.02$ & $+9.5 \pm 0.1$ & $+9.5 \pm 0.02$ & $+9.4 \pm 0.04$ & +8.4 \\
Methanol & $-17.8 \pm 0.1$ & $-18.0 \pm 0.1$ & $-18.4 \pm 0.1$ & $-18.9 \pm 0.15$ & -21.3 \\
Benzene & $-1.5 \pm 0.1$ & $-0.2 \pm 0.1$ & $+0.1 \pm 0.1$ & $-0.6 \pm 0.1$ & -3.6 \\
Phenol & $-21.7 \pm 0.02$ & $-20.1 \pm 0.2$ & $-19.7 \pm 0.1$ & $-20.2 \pm 0.1$ & -27.7 \\
Naphthalene & $-7.8 \pm 0.1$ & $-5.5 \pm 0.1$ & $-5.0 \pm 0.1$ & $-5.9 \pm 0.2$ & -9.6 \\
Anthracene & $-13.2 \pm 0.2$ & $-10.1 \pm 0.1$ & $-9.2 \pm 0.1$ & $-10.7 \pm 0.1$ & -17.7 \\
\hline
\end{tabular}

Table S2 - Solvent accessible surface area (SASA) values for the different solutes.

\begin{tabular}{cccccc}
\hline Solute & SASA $^{1}\left(\mathrm{~nm}^{2}\right)$ & Solute & SASA $\left(\mathrm{nm}^{2}\right)$ & Solute & SASA $\left(\mathrm{nm}^{2}\right)$ \\
\hline Methane & $1.43(6)$ & Methanol & $1.63(0)$ & Benzene & $2.44(1)$ \\
Ethane & $1.81(7)$ & Ethanol & $1.97(9)$ & Naphthalene & $3.10(3)$ \\
Neopentane & $2.60(7)$ & Neopentanol & $2.58(3)$ & Anthracene & $3.76(4)$ \\
Pentane & $2.75(0)$ & Phenol & $2.70(5)$ & Pyrene & $3.87(3)$ \\
Heptane & $3.36(3)$ & & & & \\
\hline
\end{tabular}

${ }^{1}$ Solvent sphere radius $1.4 \AA$.

Table S3 - Generalized parameters for the temperature dependence of $T \Delta S_{\text {hyd }}\left(\mathrm{kJmol}^{-1}\right)$, normalized by the SASA $\left(\mathrm{nm}^{2}\right)$, for aliphatic and aromatic hydrocarbons. The equation is of the form $T \triangle S / S A S A=A+B T+C T^{2}$, where $T$ is the temperature in $\mathrm{K}$.

\begin{tabular}{cccc}
\hline & $\mathrm{A}$ & $\mathrm{B}$ & $\mathrm{C}$ \\
\hline Aliphatic & 0.0185382 & -0.123008 & 0.000278762 \\
Aromatic & 0.00437478 & -0.100095 & 0.000211814 \\
\hline
\end{tabular}

Table S4 - Mean tetrahedrality, $\langle q\rangle$, of different water populations at $298 \mathrm{~K}$ and $498 \mathrm{~K}$. The no interstitial water molecules (NIWN) population includes water molecules surrounded by no more than 4 water neighbors up to $3.7 \AA$. The interstitial water molecules (IWN) population includes water molecules surrounded by 5 water neighbors up to $3.7 \AA$. The 4 or more water neighbors (4MWN) population includes water molecules in the first coordination sphere of neopentane that retain four or more water neighbors, closer than any atom of the solute.

\begin{tabular}{ccc}
\hline & \multicolumn{2}{c}{$\langle q\rangle$} \\
\hline Water Population & $298 \mathrm{~K}$ & $498 \mathrm{~K}$ \\
\hline Bulk & 0.668 & 0.477 \\
Bulk - NIWM & 0.717 & 0.443 \\
Bulk - IWM & 0.662 & 0.489 \\
Neopentane - 4MWN & 0.697 & 0.476 \\
\hline
\end{tabular}


Table S5 - Temperature dependence of the number of water molecules in the first hydration shell, $N_{\mathrm{HSh}}$, and the respective number of water molecules with four or more water neighbors (4MWN), $N_{4 \mathrm{MwN}}$, for different solutes. The last column gives the respective differences at $298 \mathrm{~K}$ and $498 \mathrm{~K}$.

\begin{tabular}{ccccc}
\hline & $298 \mathrm{~K}$ & $398 \mathrm{~K}$ & $498 \mathrm{~K}$ & $\Delta(298 \mathrm{~K}-498 \mathrm{~K})$ \\
& $N_{\mathrm{HSh}}\left[N_{4 \mathrm{MWN}}\right]$ & $N_{\mathrm{HSh}}\left[N_{4 \mathrm{MWN}}\right]$ & $N_{\mathrm{HSh}}\left[N_{4 \mathrm{MWN}}\right]$ & $\Delta N_{\mathrm{HSh}}\left[\Delta N_{4 \mathrm{MWN}}\right]$ \\
\hline Methane & $20.3[14.5]$ & $18.7[12.5]$ & $15.8[9.9]$ & $4.5[4.6]$ \\
Methanol & $20.3[13.3]$ & $18.7[11.3]$ & $16.2[9.0]$ & $4.1[4.3]$ \\
Neopentane & $25.9[14.2]$ & $22.2[10.4]$ & $17.7[7.0]$ & $8.2[7.2]$ \\
Neopentanol & $25.4[13.0]$ & $22.3[9.7]$ & $18.2[6.6]$ & $7.2[6.4]$ \\
\hline
\end{tabular}



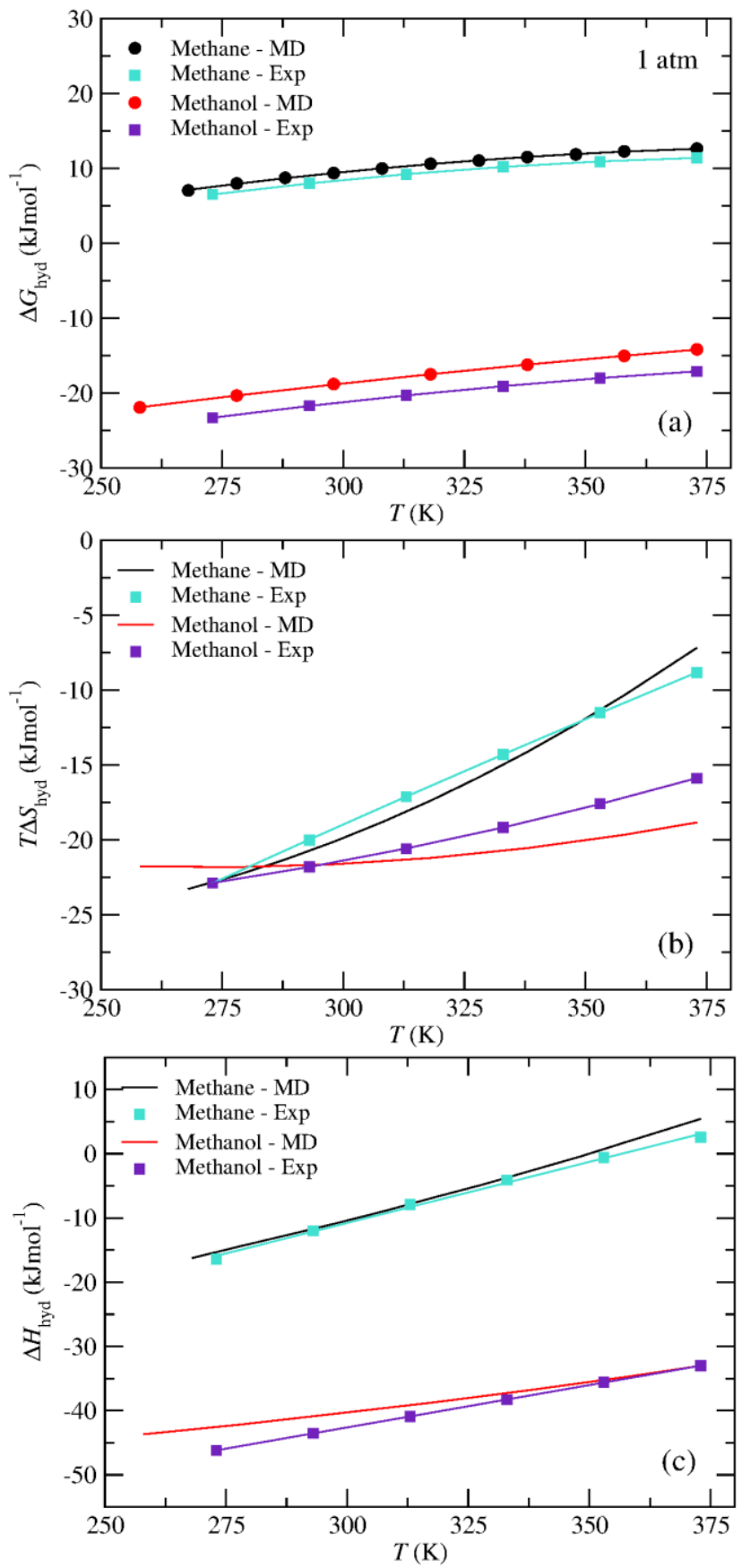

Figure S1 - Temperature dependence of the hydration (a) free energy, (b) entropy, and (c) enthalpy of methane and methanol at $1 \mathrm{~atm}$. The values from molecular dynamics (MD) simulations are compared with available "experimental" data (Table 1 of ref. 51). The methane experimental thermodynamic parameters were obtained from the experimental values at $298 \mathrm{~K}$ and $1 \mathrm{~atm}$ and the experimental $\Delta C_{p}$ up to $323 \mathrm{~K}$ and linear extrapolation of $\Delta C_{p}$ up to $373 \mathrm{~K}$. The heat capacities for methanol used to estimate the thermodynamic parameters were obtained using group additivity contributions. 

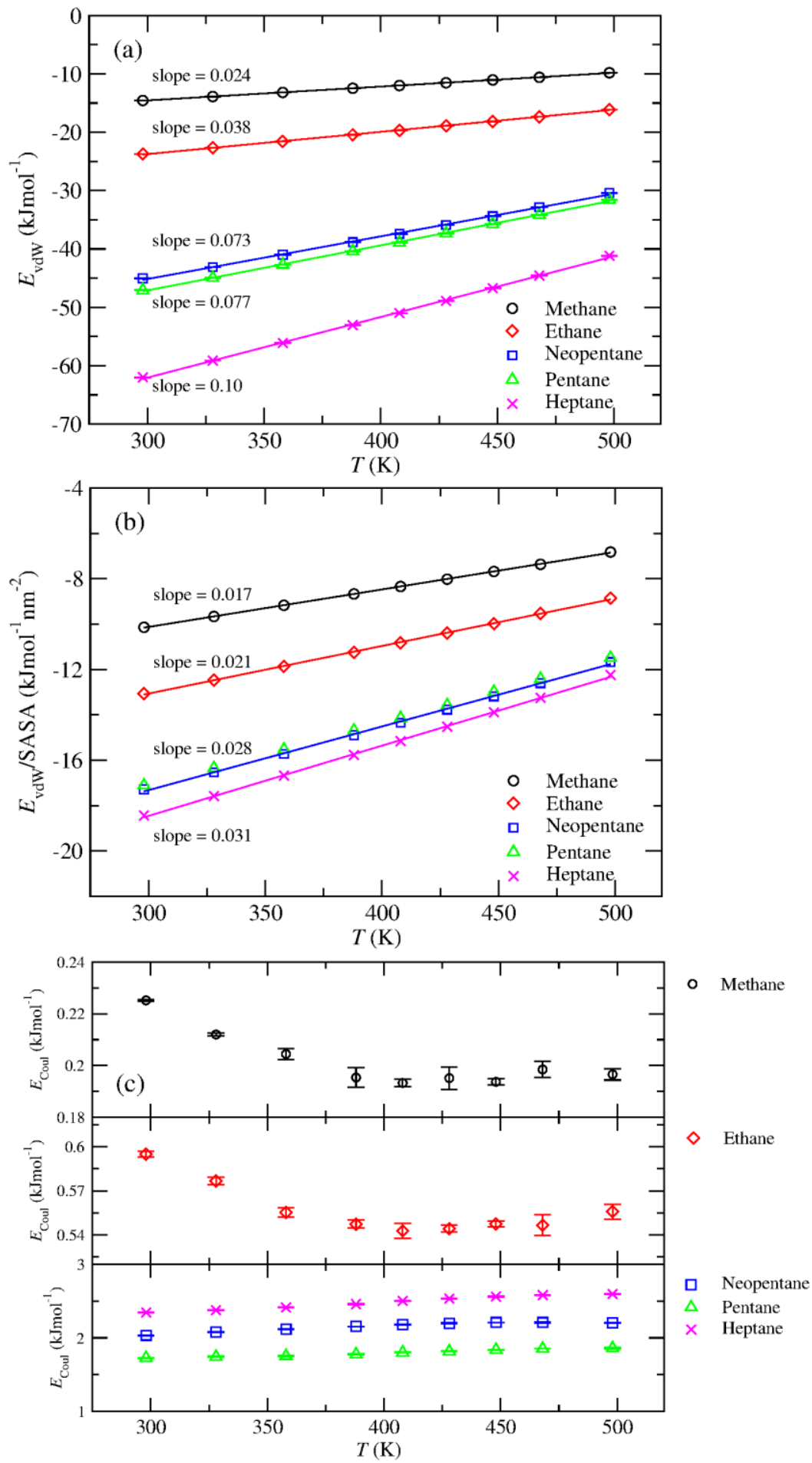

Figure S2 - Temperature dependence of the solute-water (a) van der Waals (vdW) interaction energy, (b) $\mathrm{vdW}$ interaction energy normalized by the SASA, and (c) short range $(r<10 \AA)$ component of the Coulomb interaction energy for the distinct aliphatic hydrocarbons. The rate of increase of the vdW energy with the temperature increases with the solute size. A more similar rate of increase can be observed for the van der Waals energy normalized by the SASA. The long-range contribution of the electrostatic energy is not included because the PME reciprocal space component of the electrostatic potential energy cannot be separated into solute-water and water-water components. The slopes from linear fittings (solid lines) are shown. Errors bars are standard deviations calculated from four, $20 \mathrm{~ns}$ long, independent simulations. 


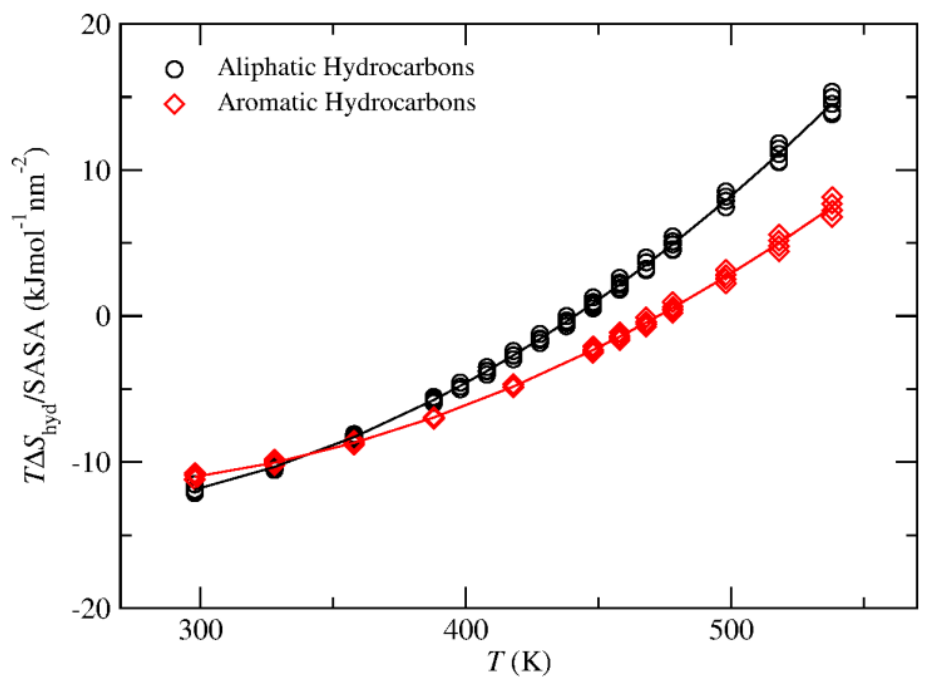

Figure S3 - Generalized (every solute) equations (solid lines) for the hydration entropy of aliphatic and aromatic hydrocarbons, normalized by the SASA. The data was fitted to a second order polynomial, $T \triangle S / S A S A=A+B T+C T^{2}$; parameters are given in Table S3. 

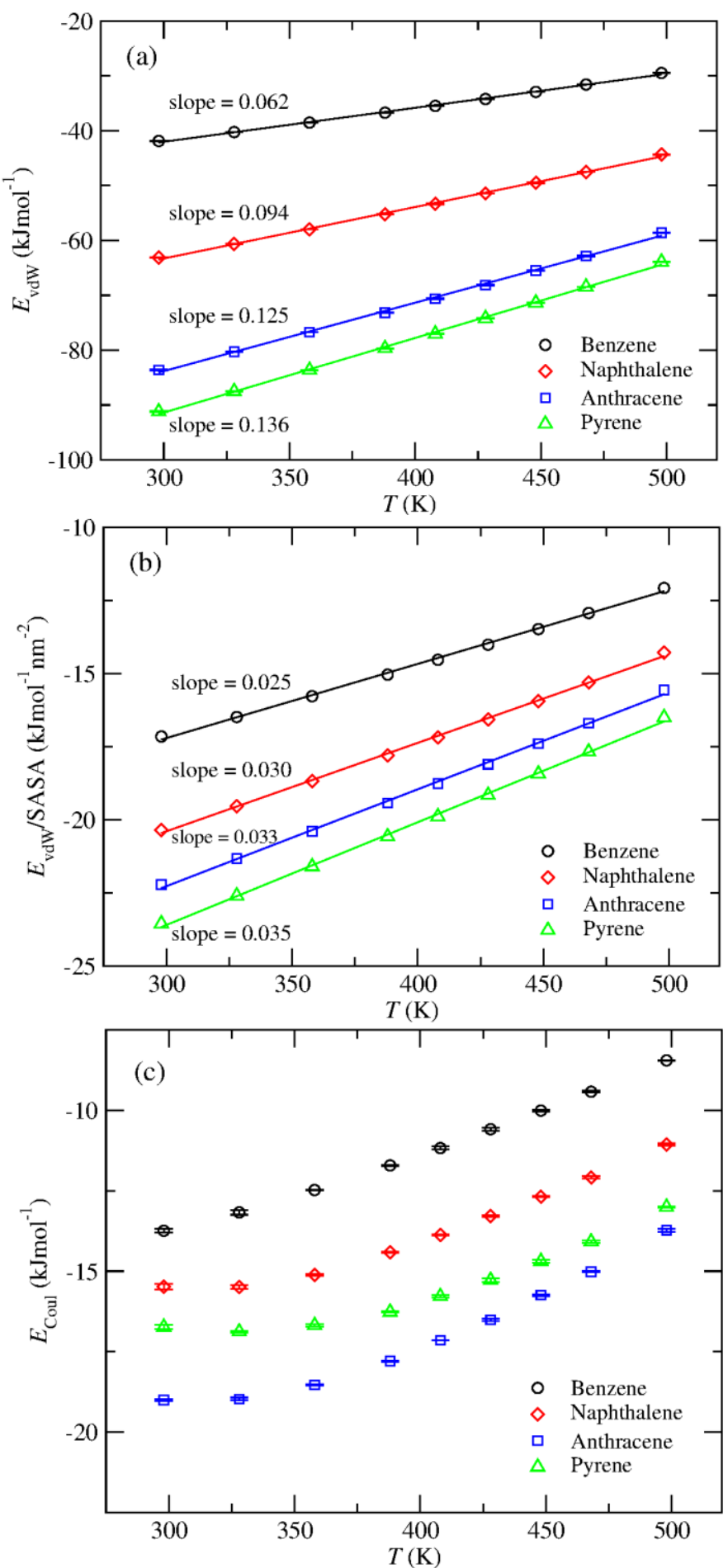

Figure S4 - Temperature dependence of the solute-water (a) van der Waals (vdW) interaction energy, (b) $\mathrm{vdW}$ interaction energy normalized by the SASA, and (c) short range $(r<10 \AA)$ component of the Coulomb interaction energy for the distinct aromatic hydrocarbons. The rate of increase of the vdW energy with the temperature increases with the solute size. A more similar rate of increase can be observed for the van der Waals energy normalized by the SASA. The slopes from linear fittings (solid lines) are shown. Errors bars are standard deviations calculated from four, $20 \mathrm{~ns}$ long, independent simulations. 

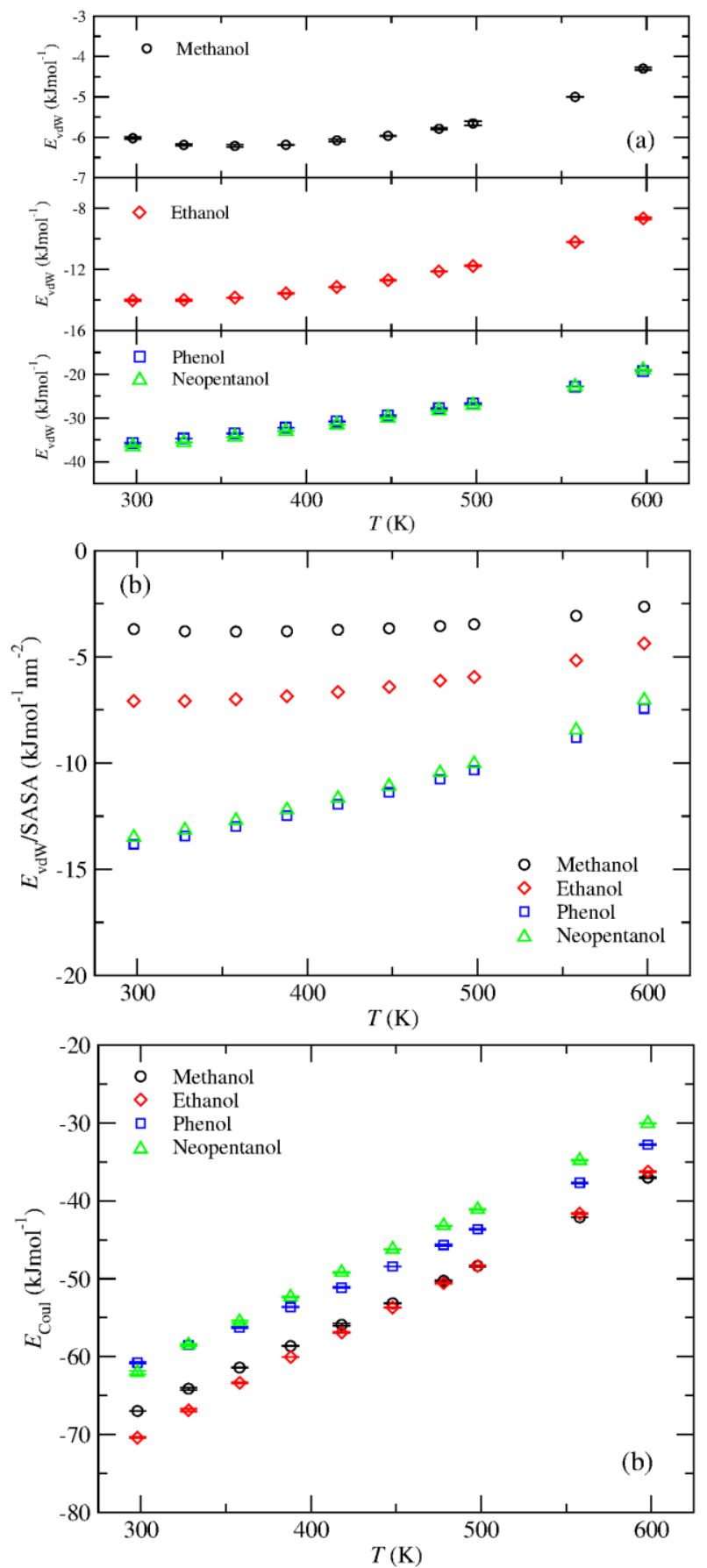

Figure S5 - Temperature dependence of the solute-water (a) van der Waals (vdW) interaction energy, (b) $\mathrm{vdW}$ interaction energy normalized by the SASA, and (c) short range $(r<10 \AA)$ component of the Coulomb interaction energy for the distinct alcohols. Errors bars are standard deviations calculated from four, $20 \mathrm{~ns}$ long, independent simulations. 


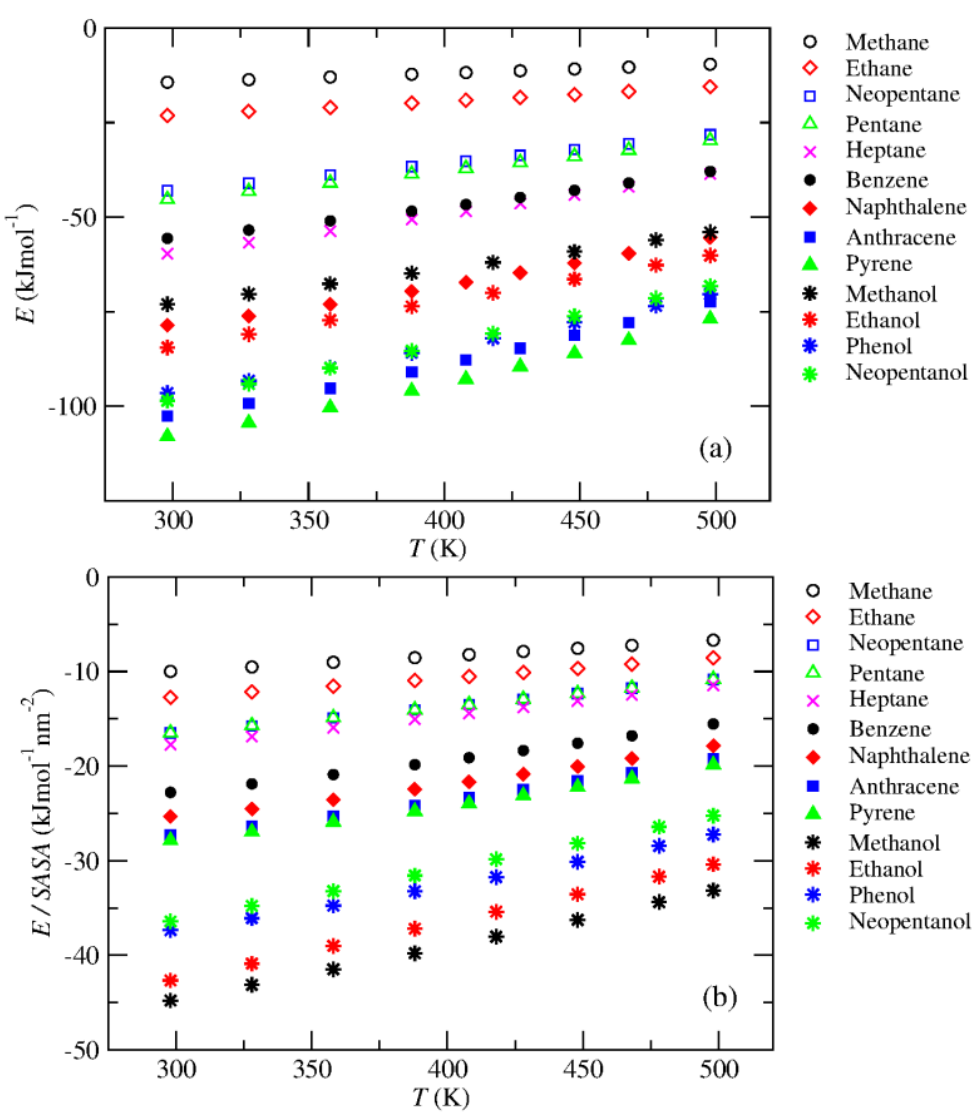

Figure S6 - Temperature dependence of the solute-water, (a) van der Waals and short-range component of the Coulomb interaction energy, $E$, and (b) $E$ normalized by the SASA.



Figure S7 - Tetrahedrality of bulk water populations with no interstitial water neighbors (NIWN) and with interstitial water neighbors (IWN) at $298 \mathrm{~K}$ and 1 atm and $100 \mathrm{~atm}$. The distributions are nearly indistinguishable, whereas the NIWM populations are $10 \%$ and $12 \%$, at $1 \mathrm{~atm}$ and $100 \mathrm{~atm}$, respectively. The density of TIP4P/2005 water at $298 \mathrm{~K}$ and $1 \mathrm{~atm}$ and $100 \mathrm{~atm}$, is, respectively, $0.997 \mathrm{~g} \cdot \mathrm{cm}^{-3}$ and $1.001 \mathrm{~g} \cdot \mathrm{cm}^{-3}$. 